\title{
Le récit de survivance de Serge Amisi : modalités d'adaptation textuelle et stratégies d'ajustement
}

\author{
Valérie DUSAILLANT-FERNANDES \\ University of Waterloo \\ Ontario/Canada
}

Résumé : Dans son récit de survivance, Souvenez-vous de moi, l'enfant de demain (2011), Serge Amisi, ancien enfant soldat en République démocratique du Congo de 1997 à 2001, raconte son enrôlement forcé dans les troupes rebelles de Kabila. Hybride parce qu'il brode des situations narratives sur un fond de vérité historique et personnelle, ce témoignage s'avère un espace scriptural privilégié, un des lieux possibles où la reconstruction du narrateur peut se réaliser. L'article propose, dans une première partie, d'explorer cette écriture singulière qui entremêle subtilement le réel et le fictionnel. Dans une deuxième partie, il s'agira de montrer comment Amisi convoque sa mémoire pour restituer les stratégies d'ajustement au stress qui lui ont permis de s'adapter aux conditions de vie ou de faire face aux punitions barbares tout en portant un regard enfantin sur une réalité historique déshumanisante.

Abstract: In the narrative of survival, Souvenez-vous de moi, l'enfant de demain (2011), Serge Amisi, former child soldier in the Democratic Republic of Congo from 1997 to 2011, recounts his story of forced recruitment in Kabila's rebel troops. A hybrid text that pushes the boundaries between fiction as well as historical and personal truth, this testimony turns out to be a privileged writing space where the social and psychic reconstruction of the narrator can be achieved. In the first part, the article explores Amisi's singular and powerful writing which blurs the lines between reality and fiction. In the second part, the paper demonstrates how Amisi summons his memory to restore the coping mechanisms which allowed him to adapt to the living conditions around him or to face the barbaric punishments while taking a childlike look at a dehumanizing historical reality.

Mots-clés : enfant soldat, stratégies, écriture, survivance, coping.

Keywords : child soldier, strategies, writing, survival, coping.

Nous constatons, depuis une vingtaine d'années, une mobilisation sans précédent contre le recrutement massif des enfants dans les conflits armés. De récentes études anthropologiques, sociologiques et littéraires sur l'enfant soldat entreprises par des chercheurs du monde entier prouvent l'aspect grandissant de ce fléau international ${ }^{1}$. De même, la communauté internationale, les dirigeants politiques ainsi que les organisations non-gouvernementales (ONG), comme l'UNICEF, unissent leurs efforts pour tenter de mettre un frein à cette exploitation abusive des enfants dans la guerre. Mais au cours de ces derniers mois, les conflits sur notre planète nous rappellent que ce phénomène d'asservissement n'est pas près de se terminer. Au Congo, au Sri Lanka, au Cambodge ou au Rwanda hier, au Soudan, en Irak ou en Centrafrique aujourd'hui, l'enfant soldat fait la une des journaux parce

\footnotetext{
1 Voir entre autres les textes suivants: Pignot, Manon (dir.). L'enfant combattant XIX-XXI siècles. Paris: Armand Colin, 2012; Bodineau, Sylvie. Figures d'enfants soldats: puissance et vulnérabilité. Laval : Presses de l’Université de Laval, 2012.
} 
que, selon Sylvie Bodineau, l'association «entre les représentations d'innocence et de vulnérabilité rattachées à l'enfance et celles de violence et d'horreur immorale rattachées à la guerre et liées à l'activité de soldat et au monde militaire est devenue inacceptable, intolérable » $(2012,40)$.

Ce recrutement des jeunes, qui n'est pas nouveau, car l'histoire nous a montré que de tout temps l'adulte a su exploiter leur innocence et leur obéissance, est tellement ravageur et universel à l'époque contemporaine qu'une définition de l'enfant soldat a été créée en 1997 et adoptée dans les Principes du $\mathrm{Cap}^{2}$, permettant ainsi d'avoir une appellation plus large et universelle sur ce que l'on entend par enfant soldat, terme jusque-là assez vague et mal défini. Ainsi est enfant soldat tout jeune de moins de 18 ans, garçon ou fille, recruté(e) volontairement ou non dans une force armée, et qui au sein de ce groupe armé peut être simple porteur d'eau, objet sexuel du leader ou combattant ${ }^{3}$. De plus, en 2002, le Protocole facultatif à la Convention relative aux droits de l'enfant concernant la participation des enfants aux conflits armés est entré en vigueur interdisant l'enrôlement des moins de 18 ans. Pourtant, plusieurs pays n'adhèrent toujours pas au Protocole. Or, si les politiques semblent fermer les yeux devant les problèmes, les victimes prennent de plus en plus la plume depuis les années 2000 pour témoigner de leur enfance volée ou violée.

Dans la littérature d'Afrique subsaharienne francophone, le sujet qui nous intéresse ici, la figure de l'enfant soldat fait l'objet d'un traitement varié aujourd'hui dans un corpus génériquement vaste comprenant des mémoires, des romans, des pièces de théâtre, des livres pour la jeunesse, des essais, des récits testimoniaux dans lesquels nous incluons les récits de survivance comme celui de Serge Amisi. Dans Souvenez-vous de moi, l'enfant de demain (2011), Amisi, ancien enfant soldat en République démocratique du Congo de 1997 à 2001, retrace son enrôlement forcé dans les troupes rebelles de Laurent-Désiré Kabila, son quotidien dans l'enfer des kalachnikovs ainsi que l'engrenage de la violence et de la drogue. Hybride parce qu'il brode des situations narratives sur un fond de vérité historique et personnelle, Souvenez-vous de moi, l'enfant de demain, également sous-titré "carnets d'un enfant de la guerre », s'avère un espace scriptural privilégié, un des lieux possibles où la reconstruction du narrateur peut se réaliser. C'est ainsi qu'Amisi livre à partir des différentes

${ }^{2}$ Ces principes ont été adoptés au Symposium sur la Prévention du recrutement d'enfants dans les forces armées et sur la démobilisation et la réinsertion sociale des enfants soldats en Afrique. Le symposium s'est déroulé au Cap (Afrique du Sud) du 27 au 30 avril 1997.

${ }^{3}$ Cette définition plus large est rédigée ainsi : «une personne, garçon ou fille, âgée de moins de dix-huit ans qui est membre, de manière volontaire ou forcée, d'une force armée (armée gouvernementale, forces armées nationales) ou d'un groupe armé (junte, armée de libération, faction armée d'un parti politique, milice). Au-delà de la fonction de combattant, un enfant soldat peut être cuisinier, porteur, coursier et toute autre personne accompagnant les groupes armés à l'exception des familles des militaires. Il peut s'agir d'une personne de sexe masculin ou féminin utilisée à des fins sexuelles ou mariée de force. L'enfant soldat n'est donc pas uniquement celui qui porte ou a porté une arme, mais celui qui, de quelque manière, est associé à une entité armée. » (Principes du Cap, 1997). 
entrées de ses carnets sa descente aux enfers, sa démobilisation et sa réinsertion par la pratique de la sculpture et du théâtre de marionnettes ${ }^{4}$. Réfugié en France où il est devenu un artiste accompli ${ }^{5}$, Amisi ne se contente pas d'exposer au grand jour le réel de nombreux autres enfants et la question de la responsabilité des groupes armés, il fait part aussi, à travers une démarche esthétique singulière, de son propre parcours face à l'adversité.

Notre analyse ${ }^{6}$ du texte d'Amisi procédera en deux parties : il s'agira, dans la première, de revenir sur certains points de la genèse du livre pour comprendre comment s'est opérée l'élaboration de la version publiée qui mélange subtilement le réel et le fictionnel. Puis, il conviendra de s'arrêter sur la forme du témoignage-carnet qui allie oralité, langue enfantine, sauts narratifs et patronymes inventés pour se protéger de toutes représailles. Après avoir examiné les modalités d'adaptation au réel d'un point de vue stylistique et narratif, notre étude proposera de mettre en évidence, dans une deuxième partie, comment Amisi convoque sa mémoire pour restituer les différentes stratégies d'ajustement au stress (" coping mechanisms ») qui lui ont permis de s'adapter assez rapidement aux conditions de vie ou de faire face aux punitions barbares tout en portant un regard enfantin sur une réalité historique déshumanisante. Notre attention se focalisera alors sur l'illusion enfantine du contrôle de l'autre et sur certaines autres stratégies de coping comme le mensonge, le jeu, la prière ainsi que les chants guerriers ou patriotiques. En fait, en se détachant du réel aux niveaux intra- et extratextuel, pour se tourner vers l'illusion et l'adaptation, Amisi s'est protégé mentalement et physiquement pour évacuer son trauma, laissant transparaitre dans ses lignes la vulnérabilité et la naïveté de l'enfant qu'il était avant son enlèvement.

\section{Récit de survivance : adaptations textuelles}

Entre 2005 et 2008, Amisi découvre l'écriture grâce à laquelle il va pouvoir continuer sa réhabilitation en se libérant de son lourd passé, revenant sur ses années passées à craindre ou à donner la mort. Dans l'entretien accordé à Patrice Yengo et Julie Peghini en 2011 à Paris, suite à la parution de son livre

\footnotetext{
4 C'est pendant ses années de démobilisation, après l'assassinat de Khabila en 2001, qu'il se reconstruit dans l'Espace Massolo, un centre de réinsertion créé en 2003 par des artistes congolais pour aider les jeunes enfants de la rue et les kadogos - terme emprunté au Swahili signifiant «petit » et qui désigne les enfants soldats - par la pratique de l'art et de l'artisanat. De retour à une vie normale au centre, il découvre le théâtre de marionnettes, un art visuel et scénique qui met en évidence l'alliance entre le corps et l'esprit, le réel et l'irréel.

${ }^{5}$ Serge Amisi continue en France, son lieu de résidence permanent, la reconstruction de son moi en se lançant dès 2010 dans la mise en scène de spectacles traversés par la danse, le chant et la manipulation de marionnettes. Du 9 au 14 février 2015, il a livré son histoire sur les planches du Théâtre de l'Échangeur à Bagnolet aux côtés du comédien Mathieu Genet et du metteur en scène Arnaud Churin.

${ }^{6}$ La publication de cet article se fait dans le cadre d'un projet de livret numérique sur le thème de l'enfant dans la littérature contemporaine d'Afrique subsaharienne francophone soutenu par le soutien financier de l'Université de Waterloo (Robert Harding Humanities and Social Sciences Award).
} 
Souvenez-vous de moi, l'enfant de demain ${ }^{7}$, on apprend qu'Amisi s'est senti poussé vers l'acte scriptural pour accompagner sa démarche artistique (107).

En fait, l'histoire de l'expérience traumatique de l'auteur est, au départ, dévoilée dans une série de huit cahiers en lingala ${ }^{8}$, soit environ mille pages écrites transcrites « au plus près de la traduction orale, sans se préoccuper $[. .$. de la "justesse" du français » remarque Jean-Christophe Lanquetin (2011, 117), l'artiste français qui a aidé Amisi à traduire son texte. Ce n'est que par la suite que l'auteur et éditeur malgache Jean-Luc Raharimanana a remanié les cahiers pour la publication des carnets en France. Ce récit livré par Amisi, victime instrumentalisée en machine de guerre, peut être qualifié de « récit de survivance », un type de récit qui fait appel au témoignage ${ }^{9}$ et qui met en scène « des sujets confrontés à des situations de crise individuelle ou sociétale » et à la perte de repères identitaires comme l'énonce Christiane Kègle (2007, 9) dans sa définition préliminaire. Selon cette spécialiste, le récit de survivance fait appel au témoignage, un genre qui « épouse plusieurs contours formels au gré des situations extrêmes dont il cherche à rendre compte » $(2007,6)$.

Au premier degré, le témoignage relève de l'oralité et «s'inscrit dans une intension résolue de dire, de raconter» (Kègle 2007, 6). Témoin conteur ${ }^{10}$, Amisi s'efforce malgré les disjonctions et les sauts narratifs dus à la forme du carnet, son instrument de travail, de rapporter les événements au passé dans une langue enfantine, laissant glisser parfois l'instantanéité du moment par des verbes conjugués au présent. Cette langue orale transposée à l'écrit dévoile une syntaxe mise à mal, une grammaire violentée, des répétitions et des contractions langagières ${ }^{11}$. Raharimanana explique que durant sa transcription, il a compris qu'Amisi était un «conteur hors pair» (Lanquetin et Raharimanana 2011, 119) et qu'il se devait de garder cette oralité d'origine tout en effectuant un travail sur la langue pour "rendre claire et acceptable la syntaxe de Serge et son rapport avec la langue » (Lanquetin et Raharimanana 2011, 124).

Ajoutons que pour Amisi, le support testimonial choisi pour transmettre son «trauma expérientiel» aux générations suivantes s'est avéré être les

\footnotetext{
${ }^{7}$ Dorénavant, les citations tirées de cet ouvrage seront suivies, entre parenthèses, par le titre abrégé Souvenez et le numéro de page.

${ }^{8}$ Dans l'entretien accordé à Yengo et Peghini, Amisi dévoile pourquoi il a écrit en lingala : «Je parle trois langues, le swahili, le kikongo et le lingala, un peu le pendé aussi. Le swahili est une langue difficile à écrire, à la différence du lingala, qu'on écrit tel qu'on le parle. » $(2011,110)$.

${ }^{9}$ Attention, il ne s'agit pas ici de témoignage historique tel que l'entend le sociologue Renaud Dulong (1998) qui demande que le témoin certifie l'exactitude et la véracité des faits.

${ }^{10}$ Nous utilisons le terme dans le sens de narrateur, celui qui conte.

${ }^{11}$ En voici quelques exemples tirés de Souvenez-vous de moi, l'enfant de demain: "Maman, comme toi tu restes ici à Kinshasa, tu seras toujours ici à Kinshasa ?» (9) ; "Et tout ce jour-là, beaucoup de gens ont eu peur de moi et ils savaient maintenant que je suis commandant. Tout ce quartier le savait. Parfois, je venais avec quatre amis, et on s'est entendus toujours la même chose, et si on vient là, tous les quatre s'éparpillent et ils me mettent au milieu comme un chef. » (37) ; «Donc on me voyait que j'étais vraiment de l'argent» (37). On note aussi la contraction du pronom « cela » en « ça » qui revient très souvent dans le récit d'Amisi.
} 
cahiers, sous-titrés "Carnets d'un enfant de la guerre » sur la couverture de son livre. Le choix du terme «Carnet» n'est pas anodin pour l'éditeur. En effet, nous notons que déjà en 1990, Louis Hay proposait une typologie sommaire des journaux, carnets et cahiers. Si le cahier se présente plus comme «le lieu de l'écriture du privé » et "se tient sur une table», le carnet, plus mobile, prend sa place dans la poche et est destiné à la prise de notes, de renseignements (1990, 9-10). De plus, Hay préfère le nom de «carnet d'esquisses » au substantif "cahier », expliquant que ce type de carnet dévoile les "premiers instantanés textuels: vers, idées, expressions, épinglés sur le coup pour ne pas échapper à la mémoire» (1990, 10). Les «carnets d'esquisses » d'Amisi seraient ainsi devenus, grâce au travail de Lanquetin et Raharimanana, des "carnets composites», des objets destinés à une écriture qui mélange intimité, « événements quotidiens et projets littéraires, fragments de formes et d'idées» (Hay 1990, 12). Nous pensons aussi que l'éditeur a pu aisément profiter du mariage entre le terme « carnet» à « enfant de la guerre », car tels les survivants de la Grande Guerre, Amisi propose un témoignage de guerre (avec ses conflits, ses actants, ses déplacements au sol) et un récit de survivant lié à l'épreuve de la perte : perte de la famille, du lien social, perte de repères, perte de l'innocence, perte d'identité. La forme du carnet favorise aussi un rythme oral, une fluidité narrative, une spontanéité, tout en apportant une dimension individuelle et collective au témoignage ${ }^{12}$.

$\mathrm{Au}$ second degré, les récits de survivance peuvent parfois s'écarter du genre autobiographique formel (Kègle 2007, 6) vu qu'il est parfois difficile pour celui ou celle qui raconte de tout dire sur soi. Que le sujet récitant le fasse volontairement ou non telle n'est pas la question. Pour Christiane Kègle, il s'agit plus de voir cela comme des «modalités d'adaptation au Réel » par lesquelles le sujet doit passer pour réussir à «sur-vivre, malgré l'insoutenable reniement de son être, et écrire son expérience de l'horreur, par définition irreprésentable, infigurable» $(2007,7)$. Dès lors, si Lanquetin a rectifié la grammaire et s'est permis de rajouter des virgules, Raharimanana a réorganisé les chapitres, enlevant quelques fragments «qui rendai[ent] la situation plus complexe » $(2011,124)$ ou des portions de texte pour une future publication. Il a changé l'ordre d'apparition de certaines personnes comme le père d'Amisi qui se trouve à la fin de la version publiée alors qu'elle est dans le quatrième cahier dans le récit original (Lanquetin et Raharimanana 2011, 124).

Ayant accepté tous ces changements, au début de la lecture de la version publiée, Amisi parvient subtilement à être l'honnête passeur d'un vécu antérieur qu'il désire transmettre aux autres, excellant dans l'art de brouiller les pistes et de mélanger les genres. D’emblée, il cherche à définir les frontières de son ouvrage, à exciter les paradoxes : «Ce livre est le récit de ma vie et de celle d'autres enfants qui m'ont accompagné dans ces moments difficiles, beaucoup ne sont plus là mais leurs histoires restent avec moi, et je les ai reprises sous forme de fictions ou comme si c'était ma propre histoire. » (Souvenez, 7).

12 Rappelons que dans son récit Amisi insère aussi des scènes vécues par d'autres enfants. 
Homme de théâtre, Amisi met donc en scène le vécu des autres, non pas avec la ferme intention de se cacher derrière la fiction, mais au contraire de se révéler par elle. En fait, il nous montre qu'à travers l'art scriptural, il y a moyen d'exposer le réel de tout un groupe d'enfants par l'intermédiaire d'artifices littéraires. Ainsi, s'il existe bel et bien une identité onomastique entre le personnage, l'auteur et le narrateur confirmant le pacte autobiographique ${ }^{13}$, Jean-Luc Raharimanana est formel : là où il existe des écarts par rapport au genre autobiographique, c'est dans les moments où Amisi raconte la vie d'autres enfants soldats. Il précise que :

la frontière entre le récit de fiction et l'autobiographie n'est absolument pas palpable dans le récit. Il faut avoir connu Serge et parlé avec lui pour savoir que là, à tel endroit, ce n'est pas sa vie qu'il raconte, mais la vie des autres enfants-soldats qu'il a connus, mais ce que les autres ont vécu, il l'a vécu intérieurement aussi, il l'a ressenti très fort, est-ce fiction, est-ce témoignage à ce moment-là? Mais ce que je retenais surtout de ce choix, c'est la forte volonté, très forte volonté de tout dire. (Lanquetin et Raharimanana 2011, 118).

Amisi ne s'amuse pas à perdre son lecteur, il sait trop bien ce qu'est l'errance pour avoir été enfant de la rue. Il cherche avant tout à trouver un équilibre pour ne pas "revenir à cette vie, ni l'encourager, ni la reconstituer » (Souvenez, 8). D'un point de vue psychologique, nous comprenons ce besoin de se distancer du réel. Les reviviscences d'un passé traumatique pourraient nuire à la bonne continuation de son projet et sa résilience fait écho à sa résistance à une autobiographie ${ }^{14}$, genre qui le ramènerait de trop près à un passé pas si lointain que cela. De plus, la fiction est là pour combler les lacunes de sa propre histoire, comme les absences mémorielles occasionnées par les effets de la drogue ou de la peur ${ }^{15}$, allant chercher ingénieusement dans la vie des autres, ces compagnons d'armes.

Soulignons, pour finir cette partie, que le récit de survivance s'inscrit dans une démarche scripturale de reconstruction de soi et de transmission. Amisi n'a pas fini de se dire, cherchant dans l'art et l'écriture à surmonter l'expérience de l'indicible et à partager son expérience, pour «laisser», dit-il dans un entretien à Selen Demir, "une trace de ce qui s'est passé et aussi montrer aux jeunes ce que [lui], enfant, [il a] vécu et que d'autres ont vécu ou sont encore en train de vivre ailleurs » $(2011,22)$. Mais qu'a-t-il vraiment vécu et quelles techniques de survie Amisi décrit-il dans son récit? Comment a-t-il réussi à s'adapter à un contexte violent et menaçant pendant quatre ans ? C'est par l'écriture qu'Amisi révèle au grand jour ses stratégies d'ajustement au stress.

13 Dès la page introductive du récit, le narrateur-enfant déclare : "Mon nom est Serge Amisi » (Souvenez, 9).

${ }^{14}$ C'est d'ailleurs avec la voix de l'enfant qu'il était qu'Amisi préfère se révéler, se distançant davantage avec un vécu douloureux.

15 Le narrateur répète plusieurs fois qu'il était souvent sous les effets des drogues qu'on lui donnait (Souvenez, 58, 59, entre autres). 


\section{Stratégies d'ajustement ou coping}

Au premier abord, il est important de ne pas oublier que l'enfant qui est enlevé à ses parents ou enrôlé de son plein gré vit sa première crise identitaire dans son nouveau milieu militaire. Pour l'intégrer au groupe, le leader du groupe armé va lui confier une fonction soi-disant importante pour exercer une pression qui vise à ébranler son appareil psychique. Ainsi, Amisi se voit nommé «chef des Kadogos » peu de temps après son arrivée au camp. Or, si pour le groupe armé, cette stratégie se révèle un moyen efficace pour rallier le garçon à sa cause et éviter qu'il ne s'échappe du camp, pour Amisi cette nomination s'apparente à un jeu de guerre : "Chef, pour moi, c'était jouer » (Souvenez, 22). Cette illusion enfantine du contrôle de l'autre par le ludisme légitime le jeune garçon dans son rôle de leader et le conforte, puisqu'il a l'impression d'avoir un contrôle sur son propre destin, passant de la passivité de victime à un agent actif au sein du groupe. En fait, le jeu permet à l'enfant de se détacher du réel angoissant qui l'entoure.

Selon Roger Caillois « dans le jeu, l'homme s'écarte du réel. Il recherche une activité libre et qui ne l'engage qu'autant qu'il en a convenu d'avance» (1950, 207). De même, pour Émile Benveniste le jeu s'impose comme une structure qui "prend origine dans le sacré, dont il offre une image inversée et brisée » $(1947,165)$. Ainsi, le jeu découpe le sacré ${ }^{16}$, le reconstitue dans un nouvel ordre qui a divisé les liens entre signifiant et signifié.

Le jeune garçon fait abstraction des conditions du réel et l'on distingue alors un décalage par rapport aux normes imposées par le contexte. Le jeu permet ainsi à l'enfant de se retrouver dans l'espace privilégié de l'entre-deux et de l'ambigüité, transformant l'ordre établi pour échapper à une réalité cruelle, dangereuse pour son moi. Cette méthode porte ses fruits. Le jeune homme, qui semble aimer son rang de chef, domine les autres d'une façon ludique et agréable. Petit à petit, il se détache de sa vie précédente, percevant son leader comme un nouveau héros et se voyant lui-même comme un de ses fidèles lieutenants. Il faut dire que tout est fait pour les transformer en machines de guerre et on les conditionne d'abord à viser des cibles mouvantes comme par exemple les trains. C'est souvent sous l'emprise de drogues, notamment le «likaya » et le «36 oiseaux », qu'Amisi est initié au «plaisir » de tirer :

On a mis des balles dans les chambres, et quand le train passait les ponts, ça donnait un bruit d'enfer avec les coups de munitions, ça donnait un bruit comme ça, bien, donc je jouais avec mon arme, et j'étais sous les effets de la drogue likaya et des 36 oiseaux, et on avait oublié que l'arme, ça tuait. (Souvenez, 58).

Dans ce souvenir lointain et en même temps si proche, puisque le terme «comme ça » met en scène l'actualisation d'un événement du passé, Amisi

\footnotetext{
16 Pour Benveniste, si «le sacré peut se définir par l'unité consubstantielle du mythe et du rite, on pourra dire qu'il y a jeu quand on n'accomplit qu'une moitié de l'opération sacrée en traduisant le mythe seul en paroles ou le rite seul en actes » $(1947,165)$.
} 
raconte une initiation divertissante au maniement des armes, ce qui le rend insensible au bruit des balles qui peuvent entrainer la mort. Cette formation forcée et contrôlée par l'emploi des stupéfiants garantit sa docilité, scelle sa loyauté au groupe et renforce son désir de plaire aux leaders.

C'est lors de la prochaine étape de son initiation qu'Amisi, complètement drogué, doit tuer son oncle qui est venu le chercher pour le ramener au village. Novice, insouciant, il exécute l'ordre sous la menace et la peur ne sachant pas vraiment que l'arme qu'il manie va tuer l'être cher qui est devant lui (Souvenez 23). Complètement dépassé par son acte meurtrier, Amisi prend le risque inconsidéré de fuir le camp au péril de sa vie. Dès lors, il subit les répercussions physiques et psychiques de son trauma. C'est avec sa voix d'enfant meurtri en quête de tendresse qu'il expose cet acte d'agression :

Quand je suis tombé malade [après avoir tué son oncle], mes amis avaient terminé la formation, on leur avait donné des armes pour qu'ils puissent aller à la guerre, et moi je me suis enfui chez une maman. [...] Et comme j'étais malade comme ça, la maman là avait souci de moi, que je puisse rester. [...] Maman-là m'a beaucoup plu, parce qu'elle avait beaucoup d'amour pour moi. (Souvenez, 25-26).

Nous notons ici l'utilisation du terme affectif de "maman» associé à l'adverbe spatial «là » qui exprime un espace neutre et distant de la maman d'« ici», du village natal. Cette maman d'adoption pour un temps limité fait figure de confidente, donnant à l'enfant la possibilité de s'exprimer sur l'acte meurtrier qu'il vient de commettre. Réfugié dans un village où on ne le connaît pas, le narrateur-enfant n'est pas encore devenu un bourreau sanguinaire, car son surmoi individuel lutte pour ne pas s'abandonner à un Moi idéal groupal ${ }^{17}$. Étant donné qu'il met la vie de cette "maman-là » en danger parce qu'elle le protège, il décide de repartir dans le camp quitte à mourir s'il le faut. En réalité, lorsque l'enfant se résout à revenir de son plein gré dans les rangs du camp militaire, c'est une forme d'appropriation subjective, "vécue dans le cadre de l'identification à l'agresseur» (Daxhelet et Brunet 2013, 229). Tout comme le jeu de «chef » qui lui confère un pouvoir spécial au sein du groupe, le fait que l'enfant se plaît à croire qu'il maitrise la situation et qu'il décide de son sort selon ses propres conditions le rassure; s'il ne se plaçait pas en "position active rassurante» (Daxhelet et Brunet 2013, 229), il serait vite envahi par des angoisses hautement traumatiques. Il y a donc illusion sur son destin et son identité. Amisi va alors mentir à ses bourreaux pour éviter que les militaires rwandais, alliés des troupes de Kabila, n'aillent exécuter cette femme de cœur. En réponse aux soldats qui lui demandent pourquoi il a fui, il déclare que cela lui a fait plaisir de revenir pour faire ce «travail de militaire » et d'ajouter : «ça m’a soulagé qu'ils m'aient cru et qu'ils aient laissé la maman qui m'avait gardé» (Somvenez, 29). Amisi va alors utiliser cette technique du mensonge pour sauver sa peau, mais aussi la peau de ceux qu'il veut épargner.

${ }^{17}$ Nous reprenons les termes de Louis Brunet $(2007,91)$. 
Ces actes barbares et gratuits le déshumanisent, l'obligeant aussi à un processus de « désengagement identificatoire » (Brunet 2007, 93), c'est-à-dire un processus défensif qui permet de « libérer le sujet du sentiment d'obligation sociale envers les non-membres du groupe puisque le processus aboutit à la représentation d'un autre qui n'est pas "comme soi" qui permet de se désidentifier de l'autre» (Brunet 2007, 93). Ainsi, faire mal ou tuer devient normal ou banal et l'enfant n'a plus de problèmes intérieurs psychiques avec son surmoi lorsqu'il commet des actes irrépréhensibles. D'autant que le groupe, qui a une fonction initiatique, «se substitue alors en partie à l'appareil psychique individuel, agissant comme une nouvelle membrane psychique » (Brunet 2007, 91), le laissant croire qu'il est dans son bon droit de tuer. Dès lors, les jeunes soldats s'entendent pour aller faire le mal (Souvenez, 48).

La rage d'écraser l'autre de peur d'être soi-même battu, fouetté ou mis au cachot est assez présente dans le récit d'Amisi. Nous avons observé que les termes «colère » (Souvenez, 24, 47, 118, 119) et «fâché » (Souvenez, 80, 82) reviennent au début du récit testimonial du narrateur-enfant surtout lorsqu'il s'impose comme bourreau et qu'il est prêt à infliger le mal aux autres, soldats ou civils, enfants et adultes confondus. Il est conscient de sa culpabilité lorsqu'il tue son oncle ou un enfant ou quand il viole une jeune fille sous les yeux approbateurs des vieux soldats de son groupe. Mais il avoue qu'il ne faisait pas ces actes «du fond du cœur» (Souvener, 81) et qu'il ne ressentait « aucun sentiment, aucun plaisir» (Souvenez, 137). Amisi exécute les ordres de son commandant (Souvenez, 88), frappant, tuant, fouettant le "papa » d'un village avoisinant au camp militaire où il est basé au point qu'il est rebaptisé «Ange rebelle » (Souvenez, 89). Il n'aime pas au fond de lui-même cette image de redoutable guerrier qu'on lui impute, voilà pourquoi il justifie son comportement devant l'homme qu'il brutalise : «Papa, moi je viens de loin. Là où j'ai laissé mes parents, je ne sais pas à cette heure qui est en train de jouer avec eux, alors moi je n'ai pas de pitié. Et je ne suis pas un prêtre, et nous les militaires on parle souvent du mal. » (Souvenez, 88). Il est clair que l'enfant soldat excuse ses actes par le fait que, quelque part au Congo, ses parents souffrent tout autant que lui sous les mains des ennemis de Kabila. Transformé, Ange rebelle ne voit plus que par la vengeance ou l'éradication pure et simple de ceux qui pourraient lui nuire ou nuire à ceux qu'il croit défendre. La force du substantif «Papa» en début de phrase souligne le caractère juvénile du raisonnement et rappelle que sous le bourreau se cache un être dépossédé de tout.

Coupables de crimes graves, les enfants-soldats ne sont pas, pour la majorité des acteurs intentionnels du mal, on le voit bien dans le récit d'Amisi. Ce réflexe à vouloir anéantir l'ennemi du groupe, quel qu’il soit, leur donne l'illusion d'invulnérabilité, d'immortalité et d'un contrôle absolu. En fait, les jeunes recrues comprennent très vite qu'il vaut mieux blesser l'autre que d'être blessé soi-même, mieux vaut tuer que d'être tué. Selon Alcinda Honwana et Thorniké Gordadze, ces enfants-soldats sont des acteurs «tactiques» dans le sens où ils agissent violemment pour 
faire face aux conditions concrètes et immédiates de leur vie, dans le but de maximiser les circonstances créées par leur environnement militaire et violent. Leurs actions sont toutefois liées à une position de faiblesse. Ils n'ont pas de base de pouvoir et agissent sur le "territoire des autres". [...] Tant bien que mal, ils réussissent à créer leur monde, un environnement de violence politique et de terreur dans lequel ils sont obligés d'agir $(2000,75)$.

Honwana et Gordadze concluent que tous les enfants soldats sont des agents interstitiels: placés malgré eux dans «une multitude d'espaces et d'états : être simultanément enfant et adulte, victime et auteur de crime, civil et militaire, etc. » $(2000,78)$. À la lecture du récit d'Amisi, nous ressentons bien cette position interstitielle dans laquelle il se trouve.

Devenant familier ou tributaire d'un système et des lieux qui l'entourent, l'enfant soldat de dix ans est donc balloté d'un état à l'autre: celui du tortionnaire, comme nous l'avons vu, d'animateur pour remonter le moral des troupes et celui de victime. Constamment mis à l'épreuve, Amisi redoute par-dessus tout qu'on le punisse, car les châtiments sont insupportables : des centaines de coups de fouet (Somvener, 55, 106, 140, 152), des mois dans un cachot (Souvener, 102), ou encore être de garde pendant plusieurs jours « sans manger» (Souvener, 53, 128, 130). Ces punitions combinées à des exercices physiques intenses sont des rites d'initiation qui ont pour but de briser l'enfant en le maintenant dans un état de terreur constant. Ainsi, le substantif " peur» (Souvenez, 58, 59, 75, 91, 95, 108) apparaît dans les différentes entrées des carnets comme pour rappeler au lecteur qu'un enfant habite toujours dans ce corps de bourreau qui tire sur les femmes et les enfants (Souvenez, 55). De même, tout au long du récit de survivance, ce qui nous a frappée, c'est également la redondance du verbe « pleurer » (Souvener, 52, 55, 131, 140, 141, 182, 191, 235) qui montre sa peine physique et psychologique. Certains passages des carnets révèlent une grande détresse, et pour survivre Amisi se tourne vers Dieu tentant de comprendre ce qui lui arrive :

J'ai pleuré, je me suis demandé devant Dieu : “Quel péché moi j’ai commis dans ce monde pour que je me retrouve comme ça dans ces situations, comme si j’avais la dette de quelqu'un ?" [...]. Avant de dormir, j'ai commencé d'abord à prier, j’ai demandé pardon à Dieu et j'ai dit: “Seigneur, pardonnez-moi mes péchés parce que ce n'était pas ma décision et si c'était ma décision, ça ne devait pas se faire comme ça, et si j'étais parmi ces gens-là, je me trouvais seulement physiquement mais dans mon cœur, je n'étais pas d'accord avec eux car je n'avais aucune envie de tout ce qui se passait. Mais quand ça m'est arrivé de faire arrêter des civils, c'est moi qui ai fait qu'ils soient arrêtés, pardonnez votre enfant, je ne veux aucun problème. Merci seigneur, amen." (Sowvenez, 141).

Plusieurs psychologues ont remarqué que lorsque les individus sont dans des situations critiques et incontrôlables qui débordent leurs ressources, ils font appel à l'aspect religieux comme processus de coping $^{18}$ - le coping étant « les pensées et les comportements qu'un individu utilise pour gérer les demandes

18 Les premières études sur le coping ont émergé durant les années 1970-1980. 
internes et externes des situations perçues comme stressantes ${ }^{19}$ (nous traduisons) (Folkman et Moskowitz 2004, 746-747). Pour Kenneth Pargament, Harold Koening et Lisa Perez (2000, 521), une des cinq fonctions ${ }^{20}$ de la religion est celle de la recherche de la signification. Un individu s'interroge sur le sens de ce qui se passe autour de lui, sollicitant les intentions de Dieu : sa bienveillance ou ses intentions punitives. La religion offre également un sentiment de confort intérieur en réduisant «l'appréhension de l'individu à vivre dans un monde où le désastre peut frapper à tout moment ${ }^{21}$ (nous traduisons) (2000, 521). Il nous semble que pour Amisi la religion joue effectivement deux rôles importants : la construction du sens et la recherche de signification ainsi que le confort intérieur qui se caractérise par un besoin de protection et d'attachement à un être suprême. Cette intimité entre le sujet et l'esprit divin est marquée par l'emploi de formules de contact soulignées par le verbe «demander», de supplications et d'un remerciement.

Après la prière, la musique est pour le narrateur une autre manière de gérer l'incompréhensible. Vladimir Yankélévitch, dans La musique et l'ineffable, rapporte que pour Debussy la musique était faite pour l'inexprimable (1983, 92). À cette réflexion du compositeur, le célèbre musicologue et philosophe français ajoute que ce qui est indicible est «ce qui rend l'homme muet en accablant sa raison et en médusant son discours. Et l'ineffable, tout à l'inverse, est inexprimable parce qu'il y a sur lui infiniment, interminablement à dire » $(1983,93)$. Il va plus loin en soulignant que sur «l'ineffable il y a de quoi parler et chanter jusqu’à la consommation des siècles » $(1983,93)$.

Au début, Amisi chante des airs patriotiques de propagande durant sa formation "accélérée » pour devenir un militaire parmi les troupes rwandaises qui le rallient à la cause de Kabila (Sowvenez 30, 34, 39) 22. Dans ses premiers chants, l'image du chef est sur-valorisée. En effet, Kabila appelle les enfants à mourir pour leur patrie menacée par Mobutu. Puis, petit à petit, asservi, Amisi découvre que lorsqu'il est déprimé, anxieux ou triste, le chant lui redonne du courage et le fait passer de l'état d'enfant docile à l'état de meneur déterminé. Il se démarque des autres grâce à son rôle d'« animateur » (Souvenez, 147, 152) glissant dans ses carnets que lorsqu'il « faisait chanter les chansons, les soldats

19 "thoughts and behaviors that people use to manage the internal and external demands of situations that are appraised as stressful."

20 Sa quatrième fonction consiste à être un mécanisme favorisant la solidarité et l'identité sociales (Pargament, Koening et Perez 2000, 521). La religion facilite la cohésion sociale car lorsque l'individu propose son aide aux autres, il se rapproche alors du groupe social et reconstruit son identité grâce à l'autre. La religion peut enfin, c'est sa dernière fonction, soutenir un individu à amorcer des changements majeurs, notamment dans la découverte de nouvelles valeurs (Pargament, Koening et Perez 2000, 521).

21 "Religion is designed to reduce the individual's apprehension about living in a world in which disaster can strike at any moment."

22 En voici un extrait dans le récit d'Amisi :

«Nos parents avaient demandé à Kabila,/Kabila où sont partis nos enfants? / Et Kabila leur avait répondu: /Vos enfants sont partis à la guerre./Le premier qui mourra, / Et le deuxième, / Le troisième qui restera, /C'est lui qui sauvera la nation. » (Souvenez, 30). 
répondaient dans une voix haute et en plaisir» (Souvenez, 126) ou avec « vivacité et toute l'énergie du peloton » (Souvenez, 145).

En fait, plusieurs chansons entonnées par le caporal Amisi et ses troupes s'avèrent être des chants libérateurs, non pas contre l'invasion de Mobutu, mais contre un des chefs de peloton, le commandant Nzembe, un être sans merci envers Amisi et ses hommes. Si dans certaines chansons les paroles sont détournées pour insulter le commandant surtout lorsque celui-ci est dans les parages (Souvener, 126), quelques-unes le nomment clairement pour le ridiculiser devant un chef plus gradé que lui (Sonvenez, 145). Quant à la chanson inspirée de marches militaires, elle fait naître un esprit d'équipe essentiel pour aller sur le champ de bataille et tuer l'ennemi.

Nous avons relevé plusieurs entrées qui dévoilent l'importance du chant dans la vie de ces "quasi-enfant ou crypto-adultes » (Honwana et Gordadze 2000, 60): «cette chanson, [...] ça nous donnait l'envie d'aller à la guerre et d'aller éparpiller les rebelles» (Somvener, 163) ou encore «j’ai pris ma conscience qu'ici, je suis à la guerre, et directement j'ai pensé à la chanson qu'on chantait. [...] J'ai enlevé la panique et j'ai fait entrer l'esprit de guerre et je n'ai plus eu de pitié.» (Somvener, 192). Les constructions phrastiques enfantines «j'ai enlevé la panique » et «j'ai fait entrer l'esprit de guerre » montrent une volonté de contrôler des émotions qui le submergent. Autrement dit, à travers la chanson Amisi exprime une représentation refoulée de son moi souffrant.

À la mort de Laurent-Désiré Kabila, Amisi a passé quatre ans à survivre aux insultes, aux coups et aux crimes commis. Il n'est plus que l'ombre de lui-même. En fait, il ressent l'assassinat de son leader en janvier 2001 comme «une guérison» (Souvenez, 237). Juste avant l'épilogue, une de ses dernières batailles est de se faire accepter dans un hôpital pour les soldats démobilisés. Or, pour être admis, il faut être vraiment blessé et la maladie dont souffre Amisi est l'état de stress post-traumatique : «j'avais la douleur dans mon cœur, jour et nuit, et mes idées, ça me remettait toujours de revivre tout ce que je venais de vivre à la guerre» (Souvenez, 240). Faute de comprendre son mal intérieur, ses amis le surnomment «Le Grand Rêveur ». Sa réalité est bien loin des rêves agréables et il se définit non pas comme un grand Rêveur mais comme un "triste penseur» (Souvenez, 242). En proie à des visions qui le hantent (Souvenez, 240, 250), il résiste encore en se promenant seul pour chasser ses idées noires (Souvenez, 242) en se persuadant que l'unique solution est de quitter l'armée (Somvenez, 242, 245, 250).

L'ancien enfant-soldat devenu enfant de la rue dans la ville de Mbandaka s'interroge à plusieurs reprises pour trouver des réponses sur l'existence, sur son enfance arrachée, sur la mort de son oncle, sur le rejet de sa famille et la vie à venir. Nous observons d'ailleurs que dans l'épilogue, le discours du narrateur, bien loin d'être dépressif, est au contraire un examen objectif de ses actions et de sa situation transitoire de l'état d'enfant soldat à l'état d'adolescent démobilisé. En quelques pages, on retrouve des expressions comme «j'ai fait le constat» (Souvener, 242, 245) et la répétition du verbe 
«décider» (Souvenez, 245, 250). Désireux de sortir de son enfer, il fait tout pour sauver sa peau prétendant en dernier recours d'être atteint d'épilepsie pour qu'on le prenne en charge (Somvenez, 250). Dans l'espace Masolo, un centre pour les enfants démobilisés, il réapprend à vivre en société, se créant une nouvelle famille à travers la sculpture, la danse et le théâtre et plus que jamais, il est déterminé par son désir de « raconter cette histoire pour qu'on n'oublie pas l'enfance qu'on [lui] a volée » (Somvenez, 254).

Dans les entrées de ses «carnets d'un enfant de la guerre», Amisi prouve que c'est en transmettant son vécu traumatique à travers la créativité que l'on réapprend à aimer son prochain, à le respecter et à se réapproprier un moi individuel perdu. À ce propos, Régine Waintrater constate que le « traumatisme a ceci de paradoxal qu'il force le sujet à trouver des façons de le surmonter, et qu'après avoir sidéré chez lui l'activité de pensée, il le contraint à le reprendre » $(2014,300)$. Dès lors, se détachant de ses multiples espaces et états identitaires vécus durant les trois phases de sa vie (enlèvement, militarisation, démobilisation), Amisi se crée un espace de reprise et de transition créatif et fiable dans les carnets, ce qui lui permet de revisiter à son rythme son enfance volée. Si la douleur et la culpabilité demeurent, car elles ne disparaittront jamais, elles peuvent être atténuées par une démarche artistique thérapeutique. Cela dit, donner une représentation plastique et scripturale à son expérience traumatique implique un travail sur soi, une réflexion introspective nécessaire pour aller vers le chemin du rétablissement intérieur. L'hybridité générique de Souvenez-vous de moi, l'enfant de demain facilite cette introspection personnelle tout en exposant le sort d'autres enfants, de ceux restés au Congo et ailleurs.

\section{Bibliographie}

Amisi, Serge. Souvenez-vous de moi, l'enfant de demain. Carnets d'un enfant de la guerre (Traduit du lingala par l'auteur avec le concours de Jean-Christophe Lanquetin, remanié par Raharimanana). La Roque-d'Anthéron: Vents d'ailleurs, coll. « Fragments », 2011.

Benvéniste, Émile. «Le jeu comme structure ». Deucalion 2 (1947) : 161-167.

Bodineau, Sylvie. Figures d'enfants soldats: puissance et vulnérabilité. Laval: Presses de l'Université de Laval, 2012.

Bodineau, Sylvie. «Réflexions sur l'autobiographie d'un enfant soldat comme espace de performativité des droits de l'enfant». Cultures-Kairós 2014. [En ligne]. Mis en ligne le 26 novembre 2014. URL: http://revues.mshparisnord.org / cultureskairos/index.php?id=937. (Consulté le 19 mars 2015).

Brunet, Louis. «Violence et appareil psychique groupal». Topique 2 no 99 (2007) : 8795.

Caillois, Roger. L’homme et le sacré. Paris : Gallimard, Coll. «Idées », 1950.

Daxhelet, Marie-Laure et Louis Brunet. «Le vécu des enfants soldats. Cheminement psychique et transformations identitaires ». La psychiatrie de l'enfant 56.1 (2013) : 219-243. 
Demir, Selen. «Les armes miraculeuses de Serge Amisi. Entretien réalisé par Selen Demir». Le Courrier de l'UNESCO 2011: 21-22. [En ligne]. URL: http://unesdoc.unesco.org/images/0019/001937/193773f.pdf. (Consulté le 20 mars 2015).

Dulong, Renaud. Le témoin oculaire. Les conditions sociales de l'attestation personnelle. Paris : Éditions de l'École des hautes études en sciences sociales, 1998.

Folkman, Susan et Judith Tedlie Moskowitz. «Coping: Pitfalls and promise » [« Le Coping : écueils et promesse »]. Annual Review of Psychology 55 (2004) : 745-774.

Hay, Louis. «L'amont de l'écriture ». In : Louis Hay (dir.). Carnets d'écrivains. 1. Paris : Éditions du CNRS, «Coll. Textes et Manuscrits », 1990 : 7-22.

Honwana, Alcinda et Thorniké Gordadze. "Innocents et coupables. Les enfantssoldats comme acteurs tactiques ». Politique africaine 4, no 80 (2000) : 58-78.

Kègle, Christiane. Les récits de survivance. Modalités génériques et structures d'adaptation au réel. Laval : Les presses de l'université Laval, 2007.

Lanquetin, Jean-Christophe et Jean-Luc Raharimanana. «Biographie du livre: dialogue sur la genèse ». Études littéraires africaines 32 (2011) : 116-125.

Pargament, Kenneth, Harold G. Koenig, Lisa M. Perez. «The Many Methods of Religious Coping : Development and Initial Validation of the RCOPE » [« Les nombreuses méthodes du coping religieux: Développement et validation initiale du RCOPE »]. The Journal of Clinical Psychology 56 (4), (2000) : 519-543.

Waintrater, Régine. «La fonction de la créativité dans l'élaboration du traumatisme extrême ». In : Emmanuel Alloa et Stefan Kristensen (dir.). Témoignage et survivance. Genève : MetisPresses, 2014 : 289-302.

Yankélévitch, Vladimir. La musique et l'ineffable. Paris : Seuil, 1983.

Yengo, Patrice et Julie Peghini. «De la vie à l'œuvre. Entretien avec Serge Amisi ». Études littéraires africaines 32 (2011) : 105-115. 